\section{Chemotherapy can replace or delay radiotherapy in pediatric intracranial ependymoma}

Radiotherapy is an effective treatment for childhood intracranial ependymoma (IE), but its use in patients younger than 5 years of age is associated with damage to the central nervous system and an increased risk of a second malignancy. A study by Grundy et al. has examined the role of chemotherapy in children with IE.

The study enrolled 89 children with IE aged 3 years or younger at diagnosis who had not received prior drug or radiation treatment. Following surgery, the children received alternating cycles of myelosuppressive and nonmyelosuppressive chemotherapy for 1 year. Among the 80 patients without metastatic disease, 50 progressed; 34 received radiotherapy for this progression. The 5-year cumulative incidence rate of freedom from radiotherapy in those patients without metastatic disease was $42 \%$. The 3-year and 5-year event-free survival rates for these patients were $47.6 \%$ and $41.8 \%$, respectively. The overall 3-year and 5-year survival rates for nonmetastatic patients were $79.3 \%$ and $63.4 \%$, respectively. Complete resection did not result in a better outcome, and overall and event-free survival did not differ according to age at diagnosis, site of disease or histological grade. The median time to progression was 1.6 years. The post-chemotherapy 5 -year overall survival rates for the patients who received the highest $(n=23)$ and lowest $(n=32)$ relative dose intensity of chemotherapy were $76 \%$ and $52 \%$, respectively.

This study shows that radiotherapy can be delayed or avoided in children with IE without compromising survival, when these patients are treated with chemotherapy.

Original article Grundy RG et al. (2007) Primary postoperative chemotherapy without radiotherapy for intracranial ependymoma in children: the UKCCSG/SIOP prospective study. Lancet Oncol 8: 696-705

\section{Potential therapy for Ph1-ALL and CML tumors unresponsive to imatinib and/or dasatinib}

Patients with blast crisis chronic myelogenous leukemia $(\mathrm{CML})$ or adult acute lymphoblastic leukemias that express the $B C R-A B L$ oncogene (Ph1-ALL) have a poor prognosis. CML typically starts with an indolent chronic phase followed by an aggressive myeloid or lymphoid blast crisis phase, which is dependent on the constitutive kinase activity of the BCR-ABL oncoprotein.

Imatinib, a BCR-ABL kinase inhibitor, shows excellent therapeutic efficacy in chronic-phase CML patients, but most patients with moreadvanced CML or ALL do not show long-term response, or develop resistance, to imatinib and to the new generation of kinase inhibitors. Alternative treatments strategies are, therefore, required. Neviani et al. recently reported that a crucial point in blast transformation of CML is the loss of protein phosphatase 2A (PP2A) activity; they have subsequently investigated the therapeutic potential of the PP2A activator FTY720 (fingolimod) by use of BCR-ABLtransformed hematopoietic cell lines, primary bone marrow progenitors from chronic phase and blast crisis CML and Ph1-ALL patients sensitive and resistant to imatinib and/or dasatinib, and mouse models of these leukemias.

The study revealed that FTY720 induces cell death and reduces clonogenicity in myeloid and lymphoid cell lines and patient-derived progenitors that were either sensitive or resistant to imatinib and/or dasatinib, but it had no adverse effects on normal bone marrow progenitor cell growth and survival. Furthermore, pharmacological doses of FTY720 markedly suppressed the in vivo leukemogenesis driven by the wildtype and dasatinib-resistant T315I BCR-ABL oncogene kinases, without any observed toxicity in control animals. The authors conclude that rescuing PP2A tumor suppressor activity could be of great therapeutic relevance in patients with advanced-phase ALL or CML who are unresponsive to imatinib and/or dasatinib.

\footnotetext{
Original article Neviani P et al. (2007) FTY720, a new alternative for treating blast crisis chronic myelogenous leukemia and Philadelphia chromosome-positive acute lymphocytic leukemia. J Clin Invest 117: 2408-2421
}

\section{IgE levels correlate with survival in patients with multiple myeloma}

Serum levels of polyclonal $\lg G$, IgA and $\lg M$ are usually decreased in patients with multiple myeloma (MM); however, little is known about the prognostic significance of polyclonal levels of IgE in these patients. Matta et al. have recently reported data on IgE levels in patients with MM 